Review began 12/07/2021 Review ended 12/12/2021 Published 12/22/2021

() Copyright 2021

So et al. This is an open access article distributed under the terms of the Creative Commons Attribution License CC-BY 4.0. which permits unrestricted use, distribution, and reproduction in any medium, provided the original author and source are credited.

\section{Use of Baricitinib in Combination With Remdesivir and Steroid in COVID-19 Treatment: A Multicenter Retrospective Study}

Jessica M. So ${ }^{1}$, Chukwuemeka Umeh ${ }^{1}$, Steven Noriega ${ }^{1}$, Erica Stratton ${ }^{2}$, Mahendra Aseri ${ }^{3}$, Rakesh C. Gupta 4

1. Internal Medicine, Hemet Global Medical Center, Hemet, USA 2. Family Medicine, Hemet Global Medical Center, Hemet, USA 3. Data Engineering and Business Intelligence, Hemet Global Medical Center, Hemet, USA 4. Pulmonary and Critical Care Medicine, Hemet Global Medical Center, Hemet, USA

Corresponding author: Jessica M. So, jessicamso@gmail.com

\section{Abstract \\ Introduction}

Hospitalized patients infected with severe acute respiratory syndrome coronavirus 2 (SARS-CoV-2) can develop severe complications. Baricitinib, a Janus kinase (JAK) JAK1/JAK2 inhibitor used to treat rheumatoid arthritis, has been proposed to prevent intracellular uptake of SARS-CoV-2 by targeting the angiotensinconverting enzyme 2 (ACE2) receptor, suppressing cytokine storm. We evaluated the effects of baricitinib on coronavirus disease 2019 (COVID-19) patient survival.

\section{Methods}

We conducted a retrospective study of 100 COVID-19 patients hospitalized in Southern California, United States, throughout September 2021. Univariate analysis of study variables was conducted with bivariate analysis of their relationships using chi-square and t-test with p-value $<0.05$ considered significant. KaplanMeier survival analysis was performed to compare outcomes of COVID-19 patients treated with baricitinib and those that were not.

\section{Results}

Our study included a patient population with a mean age of 62 years. Twenty-four percent of our patients were admitted to the intensive care unit (ICU), $16 \%$ were placed on mechanical ventilation, and $27 \%$ were expired. Patients receiving baricitinib were more likely to be admitted to the ICU and receive concomitant remdesivir therapy. Use of baricitinib increased median survival $(p=0.045)$.

\section{Conclusion}

Baricitinib administered with remdesivir and dexamethasone was shown to increase the survival of hospitalized patients with COVID-19. More studies are required to evaluate the benefits of conjunctive therapy with baricitinib, remdesivir, and dexamethasone. Though our study shows increased survival in patients receiving therapy, our study is limited by small sample size and there was not enough data to confirm whether baricitinib therapy decreased disease progression. Further studies are required.

Categories: Internal Medicine, Infectious Disease, Public Health

Keywords: hospitalized patients, mortality, cytokine storm, baricitinib, covid-19

\section{Introduction}

Hospitalized patients infected with severe acute respiratory syndrome coronavirus 2 (SARS-CoV-2) infection can progress to severe pneumonia, acute respiratory distress syndrome, multiple organ dysfunction, septic shock, and death [1]. The cytokine release syndrome, also loosely referred to as cytokine storm, has been implicated in the sudden deterioration of disease that is caused by this highly transmissible and pathogenic coronavirus [1-4]. It is associated with the activation of immune cells and the secretion of proinflammatory cytokines such as interleukin-6 (IL-6) and tumor necrosis factor- $\alpha$ (TNF- $\alpha$ ). Agents that inhibit components of this pro-inflammatory cascade have been used as treatment options to dampen the process.

Baricitinib is one of the approved agents that was given emergency use authorization by the U.S. Food and Drug Administration (FDA) to treat hospitalized patients with coronavirus disease 2019 (COVID-19) who require oxygen supplementation $[1,5,6]$. It is originally known to have clinical benefits for the treatment of patients with moderate-to-severe rheumatoid arthritis [7,8]. Baricitinib is an oral drug that works by intracellularly inhibiting the proinflammatory signal of several cytokines by suppressing Janus kinase (JAK) JAK1/JAK2. In terms of treating COVID-19 patients, it is thought to interrupt the passage and intracellular assembly of SARS-CoV-2 into the target cells mediated by angiotensin-converting enzyme 2 (ACE2) receptor 


\section{Cureus}

as well as suppressing the cytokine storm $[1,7]$.

Despite treatment advances with remdesivir and dexamethasone, reducing mortality among hospitalized COVID-19 patients remains a crucial unmet need. New treatment options are still urgently needed to reduce the high frequency of complications and deaths. There are only a few studies that evaluated the use of baricitinib in conjunction with remdesivir and dexamethasone, which showed a significant reduction in the frequency of disease progression and reduced mortality including the COV-BARRIER study $[1,9,10]$. Baricitinib was recently used in our two hospitals starting in September 2021 based on allocation issues. Clinically, we were observing that patients on high-flow oxygen were improving and being discharged home quicker. However, there was no data to confirm whether baricitinib was decreasing the rate of disease progression and decreasing the mortality rates in our two hospitals here in Southern California. We evaluated the effects of baricitinib among hospitalized COVID-19 patients with the standards of treatment in this retrospective cohort study.

\section{Materials And Methods}

This is a retrospective cohort study of 100 patients with COVID-19 admitted to two hospitals in Southern California, United States, in September 2021. The study population includes all patients who sought care at the two facilities for COVID-19-related symptoms and were diagnosed with COVID-19 through a positive polymerase chain reaction (PCR) nasopharyngeal swab. Patients de-identified data including patients age, sex, race, body mass index (BMI), ethnicity, marital status, comorbidities, laboratory results on admission, date of admission, date of discharge, medications they received while on admission, and disposition at discharge were extracted from the electronic medical record. The two hospitals had COVID-19 teams consisting of a pulmonary and critical care specialist and hospitalists, in consultation with an infectious disease specialist and pharmacist who decided patients' level of care and treatment based on existing treatment guidelines. Every COVID-19 patient was seen by one of the members of the COVID-19 team.

We did a univariate analysis of study variables using means and percentages. We performed a bivariate analysis of the relationship of different study variables with baricitinib use using chi-square test and t-test, with a p-value of 0.05 considered significant. Finally, we did a Kaplan-Meier analysis to compare survival in COVID-19 patients that received baricitinib and those that did not. We did not perform a multivariate analysis to study the relationship between mortality and baricitinib use, and the effect of patients age, sex, BMI, race, ethnicity, marital status, comorbidities, the medication that patients received while in the hospital, and laboratory results on admission, because of the small sample size of our study. The calculations were performed using IBM SPSS Statistics version 27 (Armonk, NY: IBM Corp.). This study is part of the COVID-19 studies that received exempt status from the WIRB-Copernicus Group (WCG) institutional review board (IRB), and the IRB approval number of this study is 13410516.

\section{Results}

\section{Univariate analysis}

The study included 100 patients with COVID-19. The mean age is 62 years and ranges from 23 to 94 years. Patients' mean length of hospital stay is nine days and ranges from 0 to 38 days (Table 1 ). The mean Creactive protein (CRP) on admission is 9.3 and ranges from 0.13 to 18.6 and the mean body temperature on admission is $98.9^{\circ} \mathrm{F}$ and ranges from $96.6^{\circ} \mathrm{F}$ to $104.2^{\circ} \mathrm{F}$. Fifty-one percent of the patients were female, $80 \%$ were whites, $24 \%$ were admitted in the intensive care unit (ICU), $16 \%$ were placed on the ventilator, and $27 \%$ died (Table 2).

\begin{tabular}{|c|c|c|}
\hline Statistics & Mean & SD \\
\hline Age (years) & 61.88 & 16.65 \\
\hline Body mass index (BMI) & 30.47 & 7.45 \\
\hline Length of hospital stay (days) & 8.96 & 7.94 \\
\hline
\end{tabular}

TABLE 1: Univariate analysis of continuous variables. 


\section{Cureus}

\begin{tabular}{|c|c|c|c|}
\hline \multicolumn{2}{|l|}{ Statistics } & Frequency & Percentage (\%) \\
\hline \multirow{2}{*}{ Gender } & Female & 51 & 51 \\
\hline & Male & 49 & 49 \\
\hline \multirow{3}{*}{ Race } & Black & 10 & 10 \\
\hline & Other races & 10 & 10 \\
\hline & White & 80 & 80 \\
\hline \multirow{2}{*}{ Expired } & No & 69 & 72.6 \\
\hline & Yes & 26 & 27.4 \\
\hline \multirow{2}{*}{ Ventilator use } & No & 84 & 84 \\
\hline & Yes & 16 & 16 \\
\hline \multirow{2}{*}{ ICU } & No & 76 & 76 \\
\hline & Yes & 24 & 24 \\
\hline \multirow{2}{*}{ Bradycardia } & No & 83 & 83 \\
\hline & Yes & 17 & 17 \\
\hline \multirow{2}{*}{ Remdesivir } & No & 15 & 15 \\
\hline & Yes & 85 & 85 \\
\hline \multirow{2}{*}{ Dexamethasone } & No & 17 & 17 \\
\hline & Yes & 83 & 83 \\
\hline \multirow{2}{*}{ Baricitinib } & No & 73 & 73 \\
\hline & Yes & 27 & 27 \\
\hline
\end{tabular}

TABLE 2: Univariate analysis of categorical variables.

\section{Bivariate analysis}

In the bivariate analysis of continuous variables, length of hospital stay was significantly associated with baricitinib use. The mean length of hospital stay is 14 days for patients on baricitinib compared to seven days for those that did not receive baricitinib $(\mathrm{p}=0.001)$. There was no difference in the age, BMI, or labs on admission in those that received baricitinib and those that did not (Table 3). 


\section{Cureus}

\begin{tabular}{|c|c|c|c|c|c|}
\hline Group statistics & Baricitinib use & $\mathbf{N}$ & Mean & SD & $\mathrm{p}$-Value \\
\hline \multirow{2}{*}{ Length of hospital stay (days) } & Yes & 27 & 13.78 & 8.772 & \multirow{2}{*}{0.001} \\
\hline & No & 73 & 7.18 & 6.856 & \\
\hline \multirow{2}{*}{ Age } & Yes & 27 & 57.96 & 16.068 & \multirow{2}{*}{0.154} \\
\hline & No & 73 & 63.33 & 16.744 & \\
\hline \multirow{2}{*}{ Body mass index (BMI) $\left(\mathrm{kg} / \mathrm{m}^{2}\right)$} & Yes & 27 & 32.73 & 8.08 & \multirow{2}{*}{0.065} \\
\hline & No & 73 & 29.64 & 7.08 & \\
\hline \multirow{2}{*}{ C-reactive protein (CRP) $(\mathrm{mg} / \mathrm{dL})$} & Yes & 27 & 10.6733 & 5.34279 & \multirow{2}{*}{0.148} \\
\hline & No & 61 & 8.6938 & 6.07191 & \\
\hline \multirow{2}{*}{ Lactate dehydrogenase (LDH) (U/L) } & Yes & 27 & 458.04 & 264.298 & \multirow{2}{*}{0.107} \\
\hline & No & 48 & 366.31 & 214.962 & \\
\hline \multirow{2}{*}{ D-dimer (ng/mL) } & Yes & 27 & 1347.04 & 1514.203 & \multirow{2}{*}{0.334} \\
\hline & No & 67 & 1045.85 & 1293.399 & \\
\hline \multirow{2}{*}{ Ferritin $(\mathrm{ng} / \mathrm{mL})$} & Yes & 22 & 816.286 & 564.9326 & \multirow{2}{*}{0.925} \\
\hline & No & 32 & 800.003 & 653.8097 & \\
\hline \multirow{2}{*}{ Troponin (ng/mL) } & Yes & 27 & 0.1441 & 0.46033 & \multirow{2}{*}{0.883} \\
\hline & No & 64 & 0.1623 & 0.56983 & \\
\hline \multirow{2}{*}{ Creatine phosphokinase (CPK) (U/L) } & Yes & 26 & 185.46 & 217.475 & \multirow{2}{*}{0.361} \\
\hline & No & 40 & 290.85 & 556.218 & \\
\hline \multirow{2}{*}{ Platelet $\left(10^{3} / \mathrm{mL}\right)$} & Yes & 27 & 244.93 & 105.750 & \multirow{2}{*}{0.563} \\
\hline & No & 73 & 262.41 & 142.492 & \\
\hline \multirow{2}{*}{ White blood cell (WBC) $\left(10^{3} / \mathrm{mL}\right)$} & Yes & 27 & 10.311 & 5.2061 & \multirow{2}{*}{0.360} \\
\hline & No & 73 & 9.064 & 6.2936 & \\
\hline \multirow{2}{*}{ Creatinine (mg/dL) } & Yes & 12 & 0.8883 & 0.77222 & \multirow{2}{*}{0.318} \\
\hline & No & 32 & 1.2850 & 1.26740 & \\
\hline \multirow{2}{*}{ Thyroid-stimulating hormone $(\mathrm{TSH})(\mathrm{U} / \mathrm{mL})$} & Yes & 15 & 5.2020 & 16.85474 & \multirow{2}{*}{0.420} \\
\hline & No & 22 & 2.2282 & 2.84223 & \\
\hline \multirow{2}{*}{ Temperature on admission $\left({ }^{\circ} \mathrm{F}\right)$} & Yes & 27 & 98.726 & 1.5399 & \multirow{2}{*}{0.516} \\
\hline & No & 73 & 98.973 & 1.7287 & \\
\hline \multirow{2}{*}{ Systolic blood pressure on admission ( $\mathrm{mmHg}$ ) } & Yes & 27 & 128.56 & 23.215 & \multirow{2}{*}{0.544} \\
\hline & No & 73 & 125.05 & 26.317 & \\
\hline
\end{tabular}

\section{TABLE 3: Bivariate analysis of the relationship between continuous variables and use of}

baricitinib.

In the bivariate analysis of categorical variables, ICU admission $(p=0.017)$ and remdesivir use $(p=0.011)$ were significantly associated with baricitinib use. Patients who received baricitinib were more likely to be in ICU and all patients that received baricitinib also received remdesivir. There is no baseline difference in those who received and who did not receive baricitinib based on gender, race, mortality, ventilator use, heart rate (HR), or dexamethasone (Table 4 ). 


\section{Cureus}

\begin{tabular}{|c|c|c|c|c|}
\hline \multirow{2}{*}{ Variable } & & \multicolumn{2}{|c|}{ Use of baricitinib } & \multirow{2}{*}{ p-Value } \\
\hline & & No & Yes & \\
\hline \multirow{2}{*}{ Gender } & Male & $34(69.4 \%)$ & $15(30.6 \%)$ & \multirow{2}{*}{0.425} \\
\hline & Female & $39(76.5 \%)$ & $12(23.5 \%)$ & \\
\hline \multirow{3}{*}{ Race } & Black & $8(80 \%)$ & $2(20 \%)$ & \multirow{3}{*}{0.569} \\
\hline & Other races & $6(60 \%)$ & $4(40 \%)$ & \\
\hline & Whites & $59(73.7 \%)$ & $21(26.3 \%)$ & \\
\hline \multirow{2}{*}{ Expired } & No & $53(76.8)$ & $16(23.2 \%)$ & \multirow{2}{*}{0.137} \\
\hline & Yes & $16(61.5 \%)$ & $10(38.5 \%$ & \\
\hline \multirow{2}{*}{ Ventilator } & No & $64(76.2 \%)$ & $20(23.8 \%)$ & \multirow{2}{*}{0.1} \\
\hline & Yes & $9(56.2 \%)$ & $7(43.8 \%)$ & \\
\hline \multirow{2}{*}{ ICU } & No & $60(78.9 \%)$ & $16(21.1 \%)$ & \multirow{2}{*}{0.017} \\
\hline & Yes & $13(54.2 \%)$ & $11(45.8 \%)$ & \\
\hline \multirow{2}{*}{ Bradycardia $(H R<50)$} & No & $63(75.9 \%)$ & $20(24.1 \%)$ & \multirow{2}{*}{0.148} \\
\hline & Yes & $10(58.8 \%)$ & $7(41.2 \%)$ & \\
\hline \multirow{2}{*}{ Tachycardia $(H R>100)$} & No & $37(82.2 \%)$ & $8(17.8 \%)$ & \multirow{2}{*}{0.06} \\
\hline & Yes & 36 (65.5\%) & $19(34.5 \%)$ & \\
\hline \multirow{2}{*}{ Remdesivir use } & No & $15(100 \%)$ & $0(0 \%)$ & \multirow{2}{*}{0.011} \\
\hline & Yes & $58(68.2 \%)$ & $27(31.8 \%)$ & \\
\hline \multirow{2}{*}{ Dexamethasone use } & No & $12(70.6 \%)$ & $5(29.4 \%)$ & \multirow{2}{*}{0.806} \\
\hline & Yes & 61 (73.5\%) & $22(26.5 \%)$ & \\
\hline
\end{tabular}

TABLE 4: Bivariate analysis of the relationship between categorical variables and use of baricitinib.

HR: heart rate; ICU: intensive care unit

\section{Kaplan-Meier analysis}

Kaplan-Meier analysis shows that patients that received baricitinib have increased survival compared to those that did not receive it. The median survival for those who received baricitinib is 26 days (95\% CI: 11.740.3) while it is 14 days (95\% CI: 12.4-15.6) for those who did not receive baricitinib ( $\mathrm{p}=0.045$ ) (Figure 1). 


\section{Cureus}

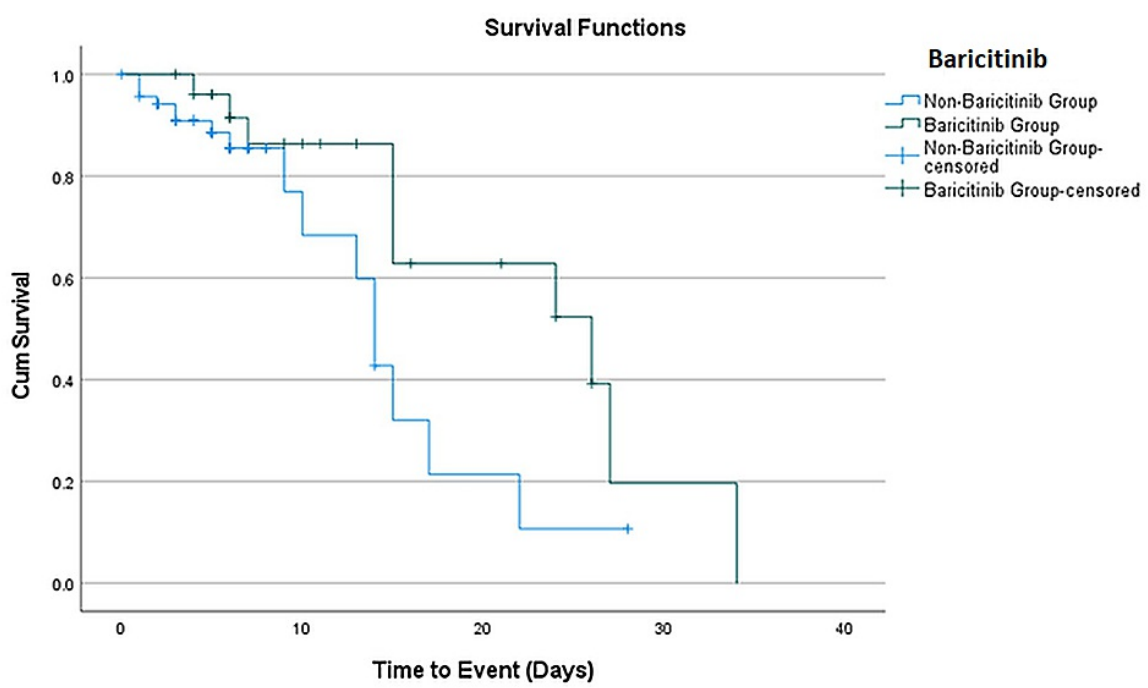

FIGURE 1: Kaplan-Meier survival curve of baricitinib use.

\section{Side effects reported with baricitinib}

Some side effects were reported in patients that received baricitinib. Thirty-three percent of the patients had a minor elevation of liver enzymes after baricitinib was started, which was not severe enough to stop the medication. The dose-adjusted baricitinib (1 mg daily) was stopped in only one patient (3.7\%) with underlying severe acute kidney injury who developed elevated liver enzymes after baricitinib was started. However, the liver enzymes returned to normal after baricitinib was stopped. Eleven percent of the patients who received baricitinib had mild creatinine elevation, $15 \%$ had transient bradycardia, and none had a fever (Table 5). Most patients (74\%) received $4 \mathrm{mg}$ of baricitinib, while $26 \%$ had their dose adjusted based on their renal function.

\begin{tabular}{|l|l|l|}
\hline Side effects reported & Frequency & Percentage (\%) (N = 27) \\
\hline Elevated liver enzymes & 9 & 33.3 \\
Elevated creatinine & 3 & 11.1 \\
Fever & 0 & 0 \\
Bradycardia & 4 & 14.8 \\
Treatment stopped due to side effect & 1 & 3.7 \\
\hline
\end{tabular}

TABLE 5: Side effects reported with baricitinib use.

\section{Discussion}

This retrospective cohort study showed that patients who received baricitinib had increased survival compared to those who did not receive the drug. The median survival for those that received baricitinib was 26 days, while it was 14 days for those that did not receive baricitinib. We considered that this was a short period to observe this small sample size of 100 patients with COVID-19, along with the fact that only $27 \%$ of these patients were treated with baricitinib. However, this survival rate benefit using this agent in conjunction with the standard of treatment with remdesivir and dexamethasone was encouraging. The patients on baricitinib in our study received an average of six days of baricitinib prior to discharge or death. Per FDA guidelines, the dosing strategy for baricitinib is $4 \mathrm{mg}$ daily for 14 days or until hospital discharge or death [6]. Additionally, a total of 27 patients who received baricitinib were also treated with remdesivir, but only 25 of those 27 patients received dexamethasone. We did not pursue a multivariate proportional hazard analysis due to our small sample size.

Limited studies are showing the benefits of combination therapy with baricitinib, remdesivir, and dexamethasone. In the COV-BARRIER study, treatment with baricitinib reduced 28-day all-cause mortality by $38.2 \%$ compared with placebo [1]. The study by Izumo et al. evaluating the clinical impact of the 
combination therapy showed that the 28-day mortality rate was low at 2.3\% (1/44 patients) and had a median hospitalization duration of 11 days and time to recovery of nine days [9]. In the ACTT-2 study, baricitinib plus remdesivir was superior to remdesivir alone in reducing recovery time and accelerating improvement in clinical status among patients with COVID-19 [11]. They showed that the 28-day mortality was $5.1 \%$ in the combination group and $7.8 \%$ in the control group [11]. Overall, these studies showed a mortality benefit with each of these agents or in combination with each other. However, we do need more randomized studies evaluating the effects of this combination therapy.

We also observed that the mean length of hospital stay was 14 days for patients on baricitinib compared to seven days for those that did not receive baricitinib $(\mathrm{p}=0.001)$. This could be attributed to starting baricitinib later during hospitalization in patients who did not improve on remdesivir and steroid therapy. It could also be explained by the fact that patients who received baricitinib were much sicker, possibly in sepsis, because those who received baricitinib in our study were more likely to be in the ICU. We observed that $45.8 \%$ of the patients who received baricitinib were more likely to be in the ICU. On an average, the ICU patients stayed longer in the hospital than non-ICU patients. At this time, we cannot differentiate between those patients who received baricitinib earlier in the course compared to those who received it later. The study by Izumo et al. showed that the median duration of ICU stay was as short as six days among patients receiving baricitinib therapy [9]. As they commented, the length of ICU stay is a critical problem in patients with severe COVID-19. We will need to do further studies to differentiate why certain patients were excluded from treatment with baricitinib.

Around $74 \%$ of the patients in our study received $4 \mathrm{mg}$ of baricitinib, while $26 \%$ had their dose adjusted based on their renal function. We also observed that $33 \%$ of the patients had a minor elevation of liver enzymes after baricitinib was started, which was not severe enough to stop the medication. Additionally, the dose-adjusted baricitinib ( $1 \mathrm{mg}$ daily) was stopped in only one patient (3.7\%) with underlying severe acute kidney injury who developed elevated liver enzymes after baricitinib was started. However, the liver enzymes returned to normal after baricitinib was stopped. The FDA guidelines recommended interrupting baricitinib if increases in the liver enzymes (alanine transaminase $\{$ ALT \} and aspartate transaminase \{AST\}) were seen. However, it did not indicate at what elevated levels to hold the medication so that drug-induced liver injury could be evaluated. In large clinical trials in rheumatoid arthritis, serum liver enzyme values were elevated in $17 \%$ of baricitinib-treated patients compared to $11 \%$ in placebo patients. These elevations were more than three times the upper limit of normal in only 1-2\% of patients on baricitinib; however, they were typically mild and transient $[12,13]$. Those studies did not report any clinically apparent liver injury associated with baricitinib [12]. Additionally, baricitinib has the potential to reactivate hepatitis B so we evaluated the status of the 27 patients in our study that received baricitinib. It was observed that $63 \%$ of our patients tested negative while the lab test was not done in $33.3 \%$ or was inconclusive in $3.7 \%$ of our patients.

Regarding adverse events, studies including the ACTT-2 study and COV-BARRIER study reviewed emergent adverse events, serious adverse events, infections, and venous thromboembolic events. They noted that the frequencies were similar between the baricitinib and placebo groups, and no new safety signals were detected $[1,11]$. We observed in our study that $11 \%$ of the patients who received baricitinib had mild creatinine elevation, $15 \%$ had transient bradycardia, and none had a fever. Though our finding is consistent with previous randomized controlled studies that showed no serious adverse effects with baricitinib, more observational studies with a large sample size are needed to report the side effects of baricitinib in clinical practice as the medication becomes more widely used in the COVID-19 treatment.

\section{Limitations of the study}

The present study had several limitations. Firstly, the study was conducted at only two centers in Southern California. Secondly, only a few patients that received baricitinib were included in the study $(\mathrm{N}=27)$, which limited our multivariate analysis ability. Lastly, our study is a retrospective observational study, and there could have been some unmeasured or unknown confounders that have influenced the outcome of our study. Despite these limitations, this study shows the positive outcomes in patients who received a combination of baricitinib, remdesivir, and dexamethasone and highlights the possible areas of future research.

\section{Conclusions}

In summary, our results show that patients that received baricitinib together with remdesivir and dexamethasone had increased survival compared to those who did not receive the drug. However, our study is limited by small sample size. Therefore, there is a need for more extensive studies to evaluate the effects of baricitinib when combined with remdesivir and dexamethasone. Furthermore, for our patients, some of them were started on baricitinib after they failed to improve on remdesivir and dexamethasone. Thus, there is a need to investigate the difference in the effect of baricitinib when started concurrently with remdesivir and dexamethasone and when started only in patients who did not improve on remdesivir and dexamethasone.

\section{Additional Information}




\section{Disclosures}

Human subjects: Consent was obtained or waived by all participants in this study. WIRB-Copernicus Group (WCG) Institutional Review Board issued approval \#13410516. WIRB-Copernicus Group (WCG) Institutional Review Board approves this study, which is part of the COVID-19 studies that received exempt status. Animal subjects: All authors have confirmed that this study did not involve animal subjects or tissue. Conflicts of interest: In compliance with the ICMJE uniform disclosure form, all authors declare the following: Payment/services info: All authors have declared that no financial support was received from any organization for the submitted work. Financial relationships: All authors have declared that they have no financial relationships at present or within the previous three years with any organizations that might have an interest in the submitted work. Other relationships: All authors have declared that there are no other relationships or activities that could appear to have influenced the submitted work.

\section{Acknowledgements}

Jessica So and Chukwuemeka Umeh contributed equally to the work and are the joint first authors of this study.

\section{References}

1. Marconi VC, Ramanan AV, de Bono S, et al.: Efficacy and safety of baricitinib for the treatment of hospitalised adults with COVID-19 (COV-BARRIER): a randomised, double-blind, parallel-group, placebocontrolled phase 3 trial. Lancet Respir Med. 2021, 9:1407-18. 10.1016/S2213-2600(21)00331-3

2. Zhang X, Zhang Y, Qiao W, Zhang J, Qi Z: Baricitinib, a drug with potential effect to prevent SARS-COV-2 from entering target cells and control cytokine storm induced by COVID-19. Int Immunopharmacol. 2020, 86:10.1016/j.intimp.2020.106749

3. Jorgensen SC, Tse CL, Burry L, Dresser LD: Baricitinib: a review of pharmacology, safety, and emerging clinical experience in COVID-19. Pharmacotherapy. 2020, 40:843-56. 10.1002/phar.2438

4. Que Y, Hu C, Wan K, et al.: Cytokine release syndrome in COVID-19: a major mechanism of morbidity and mortality. [In Press]. Int Rev Immunol. 2021, 1-14. 10.1080/08830185.2021.1884248

5. Kinase inhibitors: Janus kinase inhibitors and Bruton's tyrosine kinase inhibitors . (2021). Accessed: November 7, 2021 : https://www.covid19treatmentguidelines.nih.gov/therapies/immunomodulators/kinaseinhibitors/.

6. Fact sheet for healthcare providers. Emergency use authorization (EUA) of baricitinib . (2021). Accessed: November 7, 2021: https://www.fda.gov/media/143823/download.

7. Al-Salama ZT, Scott LJ: Baricitinib: a review in rheumatoid arthritis . Drugs. 2018, 78:761-72. 10.1007/s40265-018-0908-4

8. Richez C, Truchetet ME, Kostine M, Schaeverbeke T, Bannwarth B: Efficacy of baricitinib in the treatment of rheumatoid arthritis. Expert Opin Pharmacother. 2017, 18:1399-407. 10.1080/14656566.2017.1359256

9. Izumo T, Kuse N, Awano N, et al.: Clinical impact of combination therapy with baricitinib, remdesivir, and dexamethasone in patients with severe COVID-19. Respir Investig. 2021, 59:799-803. 10.1016/j.resinv.2021.07.004

10. Thoms BL, Gosselin J, Libman B, Littenberg B, Budd R: Efficacy of combination therapy with the JAK inhibitor baricitinib in the treatment of COVID-19. [Preprint]. Res Sq. 2021, 10.21203/rs.3.rs-835734/v1

11. Kalil AC, Patterson TF, Mehta AK, et al.: Baricitinib plus remdesivir for hospitalized adults with COVID-19. N Engl J Med. 2021, 384:795-807. 10.1056/NEJMoa2031994

12. Baricitinib. (2021). Accessed: November 11, 2021: https://www.ncbi.nlm.nih.gov/books/NBK548012/pdf/Bookshelf_NBK548012.pdf.

13. Olry A, Meunier L, Délire B, Larrey D, Horsmans Y, Le Louët H: Drug-induced liver injury and COVID-19 infection: the rules remain the same. Drug Saf. 2020, 43:615-7. 10.1007/s40264-020-00954-Z 